\title{
Un problème délicat: l'analyse de la plombémie ${ }^{1}$
}

\section{M.-N. Valloton}

Institut universitaire de médecine sociale et préventive de Lausanne (Directeur: Prof. A. Delachaux), département de médecine du travail et d'hygiène industrielle (Médecin-chef: Dr M. Lob, Prof. associé)

La plombémie, même en l'absence de tout trouble hématologique ou clinique, est considérée comme le test le plus représentatif d'une exposition au plomb. Sa détermination cependant est extrêmement délicate. Elle est effectuée à l'aide des techniques habituelles de l'analyse en trace des métaux: colorimétrie, polarographie et spectrophotométrie d'absorption atomique.

Mal appliquées ces méthodes sont susceptibles d'erreurs parfois considérables. L'auteur fait part de l'expérience acquise en ce domaine dans le département universitaire de médecine du travail et d'hygiène industrielle lors d'un travail effectué avec le professeur $R$. Hoschek de l'Office fédéral allemand pour la protection du travail à Coblence.

De cette enquête il ressort que de nombreux laboratoires appliquent des méthodes insuf-

1 Résumé d'une présentation lors de la journée d'exposés scientifiques de la Société suisse de médecine préventive, Genève, 22 juin 1972. fisamment développées: plus de $50 \%$ des laboratoires, soit ont été incapables, après 2 mois, de fournir un résultat, soit ont donné des résultats totalement erronnés.

II convient donc de recommander vivement à tout laboratoire amené à déterminer la plombémie de vérifier par des comparaisons interlaboratoires et à l'aide de méthodes standards, la précision, la spécificité et l'exactitude de sa méthode.

Il est souhaitable également que chaque médecin prenne conscience de ce genre de problème et réalise la fragilité de certains résultats sur lesquels il pourrait établir un diagnostic.

Adresse de l'auteur:

Dr M.-N. Valloton, Institut universitaire de médecine sociale et préventive, département de médecine du travail et d'hygiène industrielle, 18, César-Roux, 1005 Lausanne.

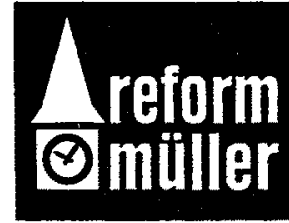

Reformhaus Müller AG B023 Zürich 1 Rennweg 15 Telefon $01256936 / 37$

\section{Diät aus dem Reformhaus Lohnende Präventivmaßnahmen Unsere Spezialitäten:}

Große Auswahl natürlicher Diät- und Kurmittel (Diabetikerprodukte, Artikel für salzlose und salzarme Diät, Leber-Galle fördernde Produkte, Kurmittel für eine gesunde und wirksame Schlankheitsdiät, Entschlackungsprodukte, Autbaumittel). 